\title{
Heritable skewed X-chromosome inactivation leads to haemophilia A expression in heterozygous females
}

\author{
Nisa K Renault ${ }^{1}$, Sarah Dyack ${ }^{2}$, Melanie J Dobson ${ }^{3}$, Teresa Costa ${ }^{4}$, Wan L Lam ${ }^{5}$ and \\ Wenda L Greer*1,6
}

\begin{abstract}
${ }^{1}$ Department of Pathology, Dalhousie University, Halifax, Canada; ${ }^{2}$ Department of Medical Genetics, IWK Health Sciences Centre and Dalhousie University, Halifax, Canada; ${ }^{3}$ Department of Biochemistry and Molecular Biology, Dalhousie University, Halifax, Canada; ${ }^{4}$ Department of Medical Genetics, Hôpital Sainte-Justine and Université de Montréal, Montréal, Canada; ${ }^{5}$ Department of Cancer-Genetics and Developmental Biology, British Columbia Cancer Research Centre, Vancouver, British Columbia, Canada; ${ }^{6}$ Department of Pathology and Laboratory Medicine, QEII Health Sciences Centre and Dalhousie University, Halifax, Canada
\end{abstract}

Factor VIII gene, F8, mutations cause haemophilia A (HA), an X-linked recessive disorder. Expression in heterozygous females has been ascribed to skewed $\mathrm{X}$-chromosome inactivation $(\mathrm{XCI})$. To investigate the cause of HA in three heterozygous females within an Atlantic Canadian kindred, the proband (severely affected girl, FVIII activity: 2\%) and 17 relatives across three generations were studied. F8 genotype, FVIII activity, $\mathrm{XCl}$ ratio $(\mathrm{XCIR})$ (paternal active $\mathrm{X}$ : maternal active $\mathrm{X}$ ), karyotype, submegabase resolution tiling set array competitive genome hybridization (competitive genomic hybridization (SMRT)), and microsatellite analyses were utilized. A positive linear relationship between FVIII activity and percentageactivated normal X-chromosome was found in HA heterozygous females $\left(R^{2}=0.87\right)$. All affected, but no unaffected females, had an XCIR skewed toward activation of the mutant X-chromosome (proband 92:8, SD 2). Unexpectedly, high numbers of females have dramatically skewed XCIRs $(>80: 20$ or $<20: 80)$ $(P<0.05)$. The distribution of XCIR frequencies within this family was significantly different than predicted by normal population data or models of random XCI $(P<0.025)$, with more females having higher degrees of skewing. Known causes of skewing, such as chromosomal abnormalities, selection against deleterious alleles, and X-inactive-specific transcript mutations, are not consistent with our results. This study shows that FVIII activity in HA heterozygous females can be directly related to XCI skewing, and that low FVIII activity in females in this family is due to unfavourable $\mathrm{XCl}$ skewing. Further, the findings suggest that these $\mathrm{XCl}$ ratios are genetically influenced, consistent with a novel heritable human $\mathrm{X}$ controlling element (XCE) functioning similarly to the mouse Xce.

European Journal of Human Genetics (2007) 15, 628-637. doi:10.1038/sj.ejhg.5201799; published online 7 March 2007

Keywords: skewed X chromosome inactivation; manifesting carrier; human XCE; factor VIII/genetics; haemophilia A/genetics

\footnotetext{
*Correspondence: Dr WL Greer, Division of Hematology, QEII Health Sciences Centre, 5788 University Avenue, McKenzie Building, Rm 223B, Halifax, Nova Scotia, Canada B3H 1 V8.

Tel: + 1902473 6691; Fax: + 1902473 4113;

E-mail: w.greer@dal.ca

Received 6 June 2006; revised 23 January 2007; accepted 31 January 2007; published online 7 March 2007
}

Introduction

Haemophilia A (HA) (OMIM 306700) is a bleeding disorder characterized by easy bruising, haemorrhage following trauma or surgery, and, in severe cases, spontaneous bleeding, especially into joints. The disease is caused by mutations in the FVIII clotting factor gene, F8. The FVIII 
protein is required for propagation of the intrinsic coagulation pathway. $\mathrm{HA}$ is an X-linked recessive disorder typically affecting hemizygous males and females homozygous for mutant F8. On occasion, heterozygous females with HA have been described, and in some cases, this has been attributed to unfavourably skewed X-chromosome inactivation $(\mathrm{XCI}) .^{1,2}$

$\mathrm{XCI}$ is a process in mammals in which one $\mathrm{X}$ in every $\mathrm{XX}$ female somatic cell is transcriptionally silenced through CpG hypermethylation and chromatin remodelling. ${ }^{3}$ Expression of the X-inactive-specific transcript (XIST) is required for silencing. ${ }^{4}$ The longstanding view has been that the determination of the X-chromosome to be inactivated (maternal or paternal) is random and independent in each cell. Females are expected to have two populations of cells: one with the maternal $\mathrm{X}$ inactivated $\left(X_{i}^{m}\right)$, and one with the paternal $X$ inactivated $\left(X_{1}^{p}\right)$. The ratio of cells with active paternal $X$ to cells with active maternal X, the XCI ratio (XCIR), should be nearly 50:50 in most females. Determination of the $\mathrm{X}$ to be inactivated has been proposed to occur within a population of eight to 16 progenitor cells. ${ }^{5}$ XCI skewing of $>80: 20$ has been considered a threshold defining a dramatically skewed phenotype. ${ }^{6}$ Random inactivation at either eight or 16 cells would be expected to result in dramatically skewed XCIR in $<9 \%$ of females. Thus, it would be unlikely to ascertain multiple females with a dramatically skewed XCIR within a small family. However, females with a dramatically skewed XCIR have been found to cluster in families. ${ }^{6}$ This suggests that XCI may not be completely random, but can be genetically influenced.

Here, we describe a family in which three males and three heterozygous females express the HA phenotype. Our objective was to determine if skewed XCIR could be related to low FVIII activity in heterozygous females and whether XCIR skewing within this family is more consistent with genetically influenced or completely random XCI.

\section{Materials and methods Participants}

The family was ascertained by the IWK Health Centre Medical Genetics Department when a young girl (proband) presented with a severe bleeding disorder at age 2.5 months (III.9 in Figure 1). Her FVIII activity was reportedly low $(0.03 \mathrm{U} / \mathrm{ml})$ and she experienced spontaneous bleeds. She was diagnosed with severe HA. She is now 3 years old and receives regular FVIII infusions. She has a 6-year-old unaffected sister. Neither her mother nor her maternal relatives have a history of HA, although her mother's maternal first cousin has mild haemophilia B (Factor IX deficiency). The proband's father and two of her paternal male first cousins have severe HA. One paternal uncle died in childhood owing to complications of HA. Her paternal grandmother and one of her paternal aunts have a history of moderate HA. They have been treated on occasion with FVIII infusions. The proband has three unaffected paternal aunts.

\section{Ethics approval}

This project (Project no. 2949) was approved by the IWK Research Ethics Board, and the Capitol Health Research Ethics Board, Halifax, Nova Scotia, Canada. Informed consent was obtained for all participants in this study.

\section{DNA extraction}

DNA from peripheral blood lymphocytes was extracted using standard techniques, ${ }^{7}$ and dissolved in TE buffer (10 mm Tris, 1 mm EDTA, pH 8.0).

\section{Cytogenetic studies}

Karyotypes were determined using methods described elsewhere. ${ }^{8}$ Bands were visualized at a resolution of $500-$ 550 .

Tiling path array competitive genomic hybridization Array competitive genomic hybridization (CGH) analysis was performed as described previously. ${ }^{9}$ Arrays (SMRT v2) were manufactured at the British Columbia Cancer Research Centre. This array spans the human physical map with 26362 BAC clones in a tiling manner. Tumour and reference DNA samples were labelled using cyanine-3dCTP and cyanine-5-dCTP, respectively, for $24 \mathrm{~h}$ at $37^{\circ} \mathrm{C}$, and purified and dissolved in DIG Easy hybridization solution (Roche Diagnostics, Laval, QC, Canada). Sample mixture was incubated with $200 \mu \mathrm{g}$ of Cot-1 DNA (Invitrogen, Carlsbad, CA, USA) for $1 \mathrm{~h}$ at $45^{\circ} \mathrm{C}$, before addition to the microarray slide. After hybridization for $48 \mathrm{~h}$, slides were washed for $5 \mathrm{~min}$ in $0.1 \times$ SSC, followed by four $5 \mathrm{~min}$ washes in $0.1 \times$ SSC with $0.1 \%$ SDS, and dried by centrifugation. Arrays were imaged using an ArrayWoRx Scanner (Applied Precision Instruments). Spot intensity ratios between the Cy3 and Cy5 channels were calculated from the resulting images using SoftWorx software, normalized for biases using CGH Norm, and visualized using SeeGH software.

\section{F8 gene intron 22 inversion analysis}

Southern hybridizations probed for F8A distinguish among non-rearranged $F 8$ genes and two common inversions, type I and type II, ${ }^{10}$ as described by Rossiter et al. ${ }^{11}$ Family members were tested for the $F 8$ gene inversion common among those with severe HA. ${ }^{12}$ The protocol was modified as described below.

Genomic DNA from peripheral blood lymphocytes from patients and controls was digested with $B c l$ I and analysed by Southern blotting and hybridization overnight at $60^{\circ} \mathrm{C}$ in $0.05 \mathrm{~mol} \mathrm{Na}_{2} \mathrm{HPO}_{4}, \mathrm{pH} 7.4,7 \%$ SDS using the 'Intron 22' probe, a $0.9 \mathrm{~kb} E c o \mathrm{RI} /$ SstI fragment of intron 22 of the $F 8$ gene. This fragment, containing $F 8 A$, was prepared from 

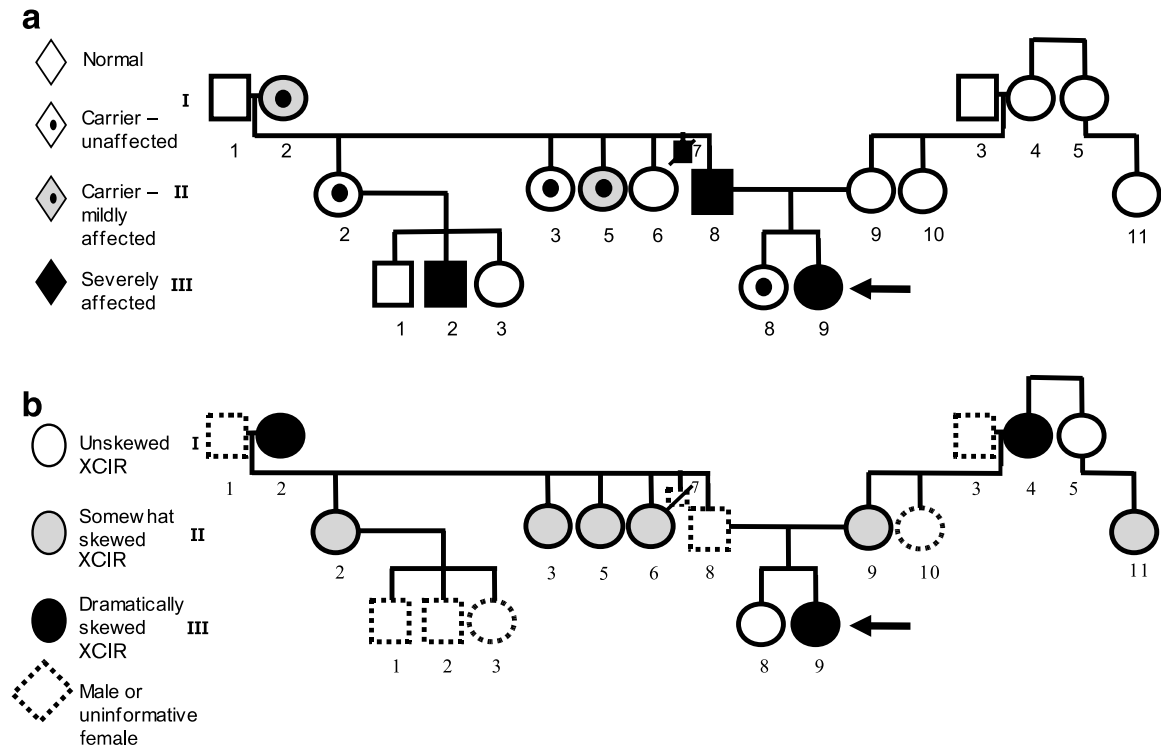

\begin{tabular}{|c|c|c|c|c|c|c|c|c|c|c|c|c|c|c|c|c|c|c|}
\hline $\mathrm{Ppt}$ & I.1 & 1.2 & 11.2 & III.1 & III. 2 & III.3 & 11.3 & 11.5 & 11.6 & \begin{tabular}{|l|}
11.8 \\
\end{tabular} & III. & |111.9 & 11.9 & II.10 & 1.3 & \begin{tabular}{|l}
1.4 \\
\end{tabular} & 1.5 & III.11 \\
\hline FVIII & - & 0.39 & 0.82 & 0.89 & 0.04 & 1.35 & 0.84 & 0.28 & 1.61 & \begin{tabular}{|c|}
$<$ \\
0.01
\end{tabular} & 0.52 & 0.02 & 1.33 & - & 1.33 & 1.61 & 0.93 & 1.05 \\
\hline F8 inv & No & $\begin{array}{l}\text { Het } \\
\text { t. II }\end{array}$ & $\begin{array}{l}\text { Het } \\
\text { t. II }\end{array}$ & No & T. II & No & $\begin{array}{l}\text { Het } \\
\text { t. II }\end{array}$ & $\begin{array}{l}\text { Het } \\
\text { t. II }\end{array}$ & No & T. II & $\begin{array}{l}\text { Het } \\
\text { t. II }\end{array}$ & $\begin{array}{l}\text { Het } \\
\text { t. II }\end{array}$ & No & No & No & No & No & No \\
\hline F8 NL & - & - & - & - & - & - & - & - & - & No & No & No & No & - & & - & & \\
\hline Age & & 72 & 48 & & & & 44 & 47 & 37 & & 5 & 2 & 33 & & & 56 & 40 & \begin{tabular}{|l|}
10 \\
\end{tabular} \\
\hline $\mathrm{XCIR}$ & - & $\begin{array}{c}18: 82 \\
\text { SD3 }\end{array}$ & $\begin{array}{l}69: 31 \\
\text { SD5 }\end{array}$ & . & - & - & $\begin{array}{c}77: 23 \\
\text { SD6 }\end{array}$ & $\begin{array}{c}39: 61 \\
\text { SD3 }\end{array}$ & \begin{tabular}{|c}
$70: 30$ \\
SD3
\end{tabular} & - & $\begin{array}{c}54: 46 \\
\text { SD3 }\end{array}$ & \begin{tabular}{|l}
$92: 8$ \\
SD2
\end{tabular} & $\begin{array}{c}30: 70 \\
\text { SD4 }\end{array}$ & - & & $\begin{array}{l}\text { 83:17 } \\
\text { SD3 }\end{array}$ & $\begin{array}{l}58: 42 \\
\text { SD2 }\end{array}$ & $\begin{array}{c}74: 26 \\
\text { SD5 }\end{array}$ \\
\hline $\begin{array}{c}F 8 \text { inv } \\
\text { on } \\
\text { active } \\
X ?\end{array}$ & - & $Y$ & $\mathrm{~N}$ & - & - & - & $\mathrm{N}$ & $Y$ & N/A & - & $\mathrm{Y}$ & $\mathrm{Y}$ & N/A & - & - & N/A & N/A & N/A \\
\hline $\begin{array}{l}\text { Most } \\
\text { active } \\
\text { X }\end{array}$ & - & $?$ & pat & - & - & - & pat & mat & pat & - & pat & pat & mat & - & - & pat & pat & pat \\
\hline
\end{tabular}

Figure 1 HA in an Atlantic Canadian family. Pedigrees highlighting the haemophilia A status (a) and XCIR skewing phenotype (b) are shown for 18 family members in three generations. (c) Molecular results are summarized. Peripheral blood samples were collected and analysed for FVIII activity (FVIII, in U/ml, ref range: $0.51-1.91 \mathrm{U} / \mathrm{ml}$ ), and F8 gene inversions (F8 inv) type I and type II. Four family members were analysed for the 6047T $>C$ mutation common in Newfoundland (F8 NL). ${ }^{13}$ Two males and one heterozygous female are severely affected, including the proband (arrow). Two heterozygous females are less severely affected. Three of the nine unaffected females are HA carriers. No females are homozygous for the $F 8$ intron 22 inversion. Average XCIR $\left(X_{a}^{p}: X_{a}^{m}\right)$ with SD from at least two independent experiments is indicated. Whether or not the most commonly active $X$ chromosome carries the F8 inversion is noted where applicable. Parent of origin (paternal, pat or maternal, mat) of the most commonly active $\mathrm{X}$ chromosome is indicated. Degree and direction of $\mathrm{XCl}$ skewing shows no parent-of-origin effect and is unrelated to $F 8$ mutation inheritance. Dramatically skewed $X C I R=>80: 20$ or $<20: 80$; somewhat skewed $X C I R=80: 20-60: 40$ or $40: 60-20: 80$; unskewed $X C I R=60: 40-40: 60$; $\mathrm{Ppt}=$ participant; Age = age (years) of participant at the time of blood collection; $\mathrm{XCIR}=$ Average $\mathrm{X}$-chromosome inactivation ratio; $\mathrm{N} / \mathrm{A}=\mathrm{no} \mathrm{F} 8$ mutation present; ' $?$ ' = phase unknown; Het t. II = heterozygous type II, ' - ' = data unavailable. Squares and circles with dashed lines indicate males, and uninformative females, respectively.

the plasmid p482.6 (ATCC cat no. 57203). To reduce nonspecific hybridization, the probe was pre-incubated for $15 \mathrm{~min}$ at $65^{\circ} \mathrm{C}$ with sheared placental DNA before use. After hybridization, blots were washed (15 min in $1 \mathrm{X}$ SSC at $60^{\circ} \mathrm{C}, 15 \mathrm{~min}$ in $0.5 \mathrm{X} \mathrm{SSC}$ at $60^{\circ} \mathrm{C}$, and $15 \mathrm{~min}$ in $0.1 \mathrm{X}$
SSC at $60^{\circ} \mathrm{C}$ ) and exposed to X-ray film (Kodak, Rochester, NY, USA) for 2 days at $-80^{\circ} \mathrm{C}$, or analysed on a GS-525 Molecular Imaging phosphorimager (Biorad Laboratories Ltd, Mississanga, ON, Canada) and quantified using Molecular Analyst Software v. 1.5 (Biorad). 
F8 Newfoundland mutation analysis

To assay for the $F 8$ mutation common in Newfoundland, Canada, $(6047 \mathrm{~T}>\mathrm{C}),{ }^{13}$ an allele-specific polymerase chain reaction (PCR) was used to selectively amplify either the normal or the mutant sequence. Primer HA19ASP1 (5'Agc AcA cTT TTT cTg gT- $\left.3^{\prime}\right)$ and primer HA19ASP2 (5'-Agc AcA cTT TTT CTg ge $\underline{\mathbf{c}}-3^{\prime}$ ) specifically bind the normal and mutant sequences, respectively. Both forward primers, the common reverse primer (E19-2: $5^{\prime}$-Agc AAc cAT Tcc AgA AAg $g A-3^{\prime}$ ) and controls were generously provided by $\mathrm{Dr}$ Yangyang Xie, director of the Molecular Genetics Laboratory, NL, Canada.

\section{Conformation sensitive gel electrophoresis}

To screen for additional $F 8$ gene mutations, samples were tested for the intron 1 inversion ${ }^{14}$ and analysed by conformation sensitive gel electrophoresis (CSGE) ${ }^{15}$ by Dr Lillicrap, National Program for Hemophilia Mutation Testing, Department of Pathology and Molecular Medicine, Kingston, Ontario. Together with the $F 8$ intron 22 inversion test, these techniques detect $85-90 \%$ of $F 8$ gene mutations (Dr Lillicrap, personal communications).

\section{Functional factor VIII activity}

Functional FVIII activity was measured using the Hemoliance Factor VIII Deficient Plasma Kit according to the manufacturer's directions (Hemoliance, product code no. 49738081). This is the standard method used at the IWK and the QEII Health Sciences centre to assess HA severity. Peripheral blood was collected over sodium citrate and made platelet poor by centrifugation. Samples were processed immediately, or frozen within $6 \mathrm{~h}$ and shipped to our lab for processing. Functional FVIII activity was determined by comparing clotting times to a standard curve. Using this protocol, the normal range of FVIII activity is $0.51-1.93 \mathrm{U} / \mathrm{ml}$.

\section{Determination of XCIRs in blood}

Digestion and PCR amplification: XCIRs $\left(X_{a}^{p} X_{a}^{m}\right)$ were determined by quantitation of the methylation status of the polymorphic human androgen receptor (HUMARA) locus as described elsewhere, ${ }^{16}$ with some modifications. The methylation sensitive restriction endonucleases, HhaI and HpaII, yield equivalent results in this assay. ${ }^{5}$ We chose Hha I because it more consistently digested to completion in our hands. DNA samples from each participant $(0.7 \mu \mathrm{g}$ DNA) and controls (water) were incubated for $5 \mathrm{~h}$ at $37^{\circ} \mathrm{C}$ with or without HhaI under appropriate digestion conditions (Invitrogen). Reactions were terminated by incubation at $95^{\circ} \mathrm{C}$ for $10 \mathrm{~min}$. Approximately, $0.1 \mu \mathrm{g}$ was used as a template for PCR using $10 \mathrm{pmol}$ of primer hetero $1\left(5^{\prime}\right.$ gcTgTgAAggTTgcTgTTccTcAT-3') and of primer hetero 2 $\left(5^{\prime} \text {-TccAgAATcTgTTccAgAgcgTgc- } 3^{\prime}\right)^{17}$ in $1 X$ PCR Rxn buffer (Invitrogen), $1.5 \mathrm{mM} \mathrm{MgCl}_{2}$ (Invitrogen), $0.2 \mathrm{mM} \mathrm{dNTP}$ (Roche), $1 / 30 \mathrm{v} / \mathrm{v}$ DMSO, $1.25 \mathrm{U}$ Taq DNA polymerase
(Invitrogen), $1 \mu \mathrm{Ci} \quad\left[\alpha^{32} \mathrm{P}\right]-\mathrm{dCTP} \quad$ (GE Healthcare, BioSciences Inc., Baie d'Urfé, QC, Canada). Samples were amplified using a PTC-100 programmable thermal controller thermocycler (MJ Research Inc., Waltham, MA, USA) by denaturation $\left(95^{\circ} \mathrm{C}\right.$ for $\left.5 \mathrm{~min}\right)$, followed by 28 cycles of $95^{\circ} \mathrm{C}$ for $45 \mathrm{~s}, 60^{\circ} \mathrm{C}$ for $30 \mathrm{~s}$, and $72^{\circ} \mathrm{C}$ for $30 \mathrm{~s}$, followed by a final extension $\left(7 \mathrm{~min}\right.$ at $\left.72^{\circ} \mathrm{C}\right)$.

XCIR determination: PCR products were resolved on $6 \%$ denaturing polyacrylamide gels (1:19 N, $N^{\prime}$-methylenebisacrylamide: acrylamide (Invitrogen); 9M Urea; $1 \mathrm{X}$ TBE buffer) at $45^{\circ} \mathrm{C}$ for $5 \mathrm{~h}$ at $80 \mathrm{~W}$. The abundance of each HUMARA allele PCR product was determined by phosphorimager analysis. Some alleles amplified more efficiently than others. To allow comparisons to be made, the ratio of amplification products obtained for the two alleles after Hhal digestion was normalized relative to the ratio without digestion. The PCR procedure amplifies inactive (ie methylated) HUMARA alleles, thus the ratio of amplification products (ie intensity of paternal allele: intensity of maternal allele) is the inverse of XCIR, which is described in terms of active $\mathrm{X}$ chromosomes $\left(\mathrm{X}_{\mathrm{a}}^{\mathrm{p}}: \mathrm{X}_{\mathrm{a}}^{\mathrm{m}}\right)$. In informative females, it was possible to determine which allele was paternally derived or maternally derived by comparing allele sizes between parents and their children or between sisters. Each XCIR was calculated as an average of at least two replicate assays. Using the established threshold, ${ }^{6}$ a female was considered to have a dramatically skewed XICR if her average XCIR was $>80: 20$ or $<20: 80$. We considered an XCIR somewhat skewed, or unskewed if the average XCIR fell between $80: 20$ and $60: 40$ or $40: 60$ and 20:80, or between $60: 40$ and 40:60, respectively.

\section{Microsatellite analyses}

Peripheral blood DNA from the proband, her sister, and her parents were analysed at the Australian Genome Research Facility (AGRF), Victoria, Australia (www.agrf. org.au), at three microsatellite loci in the X-inactivation centre (XIC) region (DXS986, DXS1196, and DXS990 at Xq21.1, Xq21.31, and Xq21.32, respectively). Cytogenetic location of each marker was determined by the annotations in Ensembl version 35 (www.ensembl.org), physical locations as annotated on the UCSC Golden Path (http://genome.ucsc.edu/).

\section{Statistical calculations}

The $\chi^{2}$ test was used to compare the observed number of females with dramatically skewed XCI, and the distribution of frequencies of XCIRs in this family with that found in the general population, and with those predicted by models of random XCI. In the normal population examined elsewhere, ${ }^{5}$ the average XCIR was 49:51, standard deviation (SD) 17 . Only $8 \%$ of females were dramatically skewed. As XCI is thought to occur within a population of eight to 16 progenitor cells, ${ }^{5}$ two models were developed. The eight-cell model predicts an average 
XCIR of 50:50, SD 17.71, and that $8.92 \%$ of females will be dramatically skewed. These values are very similar to those of the normal population. The 16-cell model predicts an average XCIR of 50:50, SD 12.5, and that $1.64 \%$ of females will be dramatically skewed. Assuming random XCI in all three cases, 68,27 , and $5 \%$ of females would be expected to have XCIRs within $1 \mathrm{SD}$, between 1 and 2 SD, and $>2$ SD of the mean, respectively.

The percentage of lymphocytes with the normal $F 8$ allele on the active $\mathrm{X}\left(\% \mathrm{X}_{\mathrm{a}}^{\mathrm{F}}\right)$ was determined by the $\mathrm{XCIR}$ and the identification of the maternal or paternal origin of the $\mathrm{X}$ chromosome carrying the mutant $F 8$ allele in heterozygous women. The relationship between FVIII activity and $\% \mathrm{X}_{\mathrm{a}}^{\mathrm{F} 8}$ was determined by linear regression analysis.

\section{Results}

\section{Plasma factor VIII activity}

HA severity in each participant was evaluated by measuring plasma FVIII activity using a functional clotting assay. For individuals receiving FVIII infusions (II.8, III.2, III.9), relevant pre-infusion FVIII activity measurements were taken from medical records (Figure 1). As an individual's FVIII activity does not vary significantly over short to moderate time periods, ${ }^{18}$ one FVIII activity was determined for each of the remaining study participants.

The proband's FVIII activity was 0.02. Two of her female relatives (I.2, II.5), both of whom had a history of HA expression, had FVIII activity below the normal range $(0.39,0.28$, respectively). Although the proband's sister was also heterozygous for the F8 mutation, her FVIII activity was 0.52 . This is approximately half the median normal value, as would be expected in a heterozygote carrier with an XCIR of about 50:50. All homozygous wild-type female relatives and heterozygous females not expressing HA had FVIII activities within the normal range.

\section{Karyotype and tiling path array CGH}

Chromosomal abnormalities, such as balanced X:autosome translocations, can result in the exclusive survival of cells that have inactivated the normal $\mathrm{X}$ chromosome, allowing correct expression of the autosome. This can lead to the expression of $\mathrm{X}$-linked recessive disorders in heterozygous women if the derivative X-chromosome harbours a recessive disease allele or if the translocation disrupts a disease causing gene. ${ }^{19}$ We have determined by standard cytogenetic analyses that the most extremely skewed girl (III.9) has a normal karyotype (data not shown), and by tiling path array CGH (SMRT v2) at submegabase resolution that no amplifications or deletions are present (data not shown). Thus, the dramatically skewed XCIRs in this family are unlikely to be due to chromosomal abnormalities.
F8 intron 22 inversion analysis

Roughly $40 \%$ of severe haemophiliacs have chromosomal inversions involving intron 22 of the $F 8$ gene. Of these, approximately $82 \%$ have the distal inversion (type I) and $18 \%$ have the proximal inversion (type II). ${ }^{12}$ As is standard of care at the IWK Health Centre for families with severe HA, each participant was evaluated for these two F8 gene intron 22 inversions using a Southern blotting assay. ${ }^{10} \mathrm{No}$ type I inversions were detected (data not shown). Six of the paternal family members (I.2, II.2, III.2, II.3, II.5, II.8) have a type II inversion (Figure 1) (data not shown). No inversions were detected in the proband's maternal relatives. All affected males and females have a single copy of the type II inversion, as do obligate carriers. No female has more than one inverted allele. Our controls included one homozygous normal individual and one male with a type I inversion.

\section{F8 NL mutation analysis}

As the proband has ancestry that can be traced back to NL on both her maternal and paternal sides, the proband, her sister, and her parents were tested for the $F 8$ mutation common in NL (disease prevalence of 44/3300 males in some NL communities ${ }^{13}$ ). This mutation, $6047 \mathrm{~T}>\mathrm{C}$, is a missense mutation (V2016A) causing mild HA. No NL F8 mutations were detected in any of the participants tested (Figure 1) (data not shown).

\section{Mutation screening by CSGE}

To rule out any other $F 8$ mutations, the proband and her mother were assessed for the intron 1 inversion and screened by CSGE for other mutations. No mutations were found in the proband's mother (II.9), and no additional mutations were found in the proband (III.9, data not shown).

\section{$\mathrm{XCI}$ ratios}

To assess whether affected females in this family have skewed XCI, we determined the XCIR at the HUMARA locus. The HUMARA gene contains a variable length polymorphism adjacent to an HhaI restriction endonulease cleavage site. This site is methylated exclusively on the $\mathrm{X}_{\mathrm{i}}$, thus only unmethylated $X_{a}$ alleles are subject to digestion by this methylation sensitive endonuclease. PCR amplification of this region is precluded by HhaI digestion. This assay allows the calculation of the relative inactivation of the paternal and maternal $\mathrm{X}$ chromosomes, which is the inverse of the XCIR (active paternal to maternal $\mathrm{X}$ chromosomes). A female was considered informative at this marker only if she had two alleles that consistently resolved on a $6 \%$ polyacrylamide gel. Male DNA, containing only one active $\mathrm{X}$ chromosome, is used as a positive control.

All three females with a history of HA expression and low FVIII activity (I.2, II.5, III.9) were found to have skewed 
XCI in favour of the mutant X (Figure 1). Three additional paternal aunts (II.2, II.3, II.6) with no history of HA expression or low FVIII activity also had skewed XCI. Two of these (II.2, II.3) were skewed in favour of the normal X, whereas the third (II.6) did not inherit the F8 mutation. Six out of seven informative females had somewhat or dramatically skewed XCI. The proband had the most extreme XCIR skewing (92:8 SD 2). Because of this, the possibility of an additional skewing influence from the maternal side of the family was investigated. Four out of six informative females had somewhat or dramatically skewed XCI. Again, the proband had the most extreme XCI skewing. A potentially nonrandom pattern of XCI skewing in both sides was found, so subsequent analyses of XCI skewing included both paternal and maternal relatives of the proband.

In this family, three of eleven informative females have dramatically skewed XCI (I.2, I.4, III.9). Six more are somewhat skewed (II.2, II.3, II.5, II.6, II.9, II.11). Surprisingly, only two females are unskewed (I.5, III.8). Two females were uninformative at the HUMARA locus and were not included in the analysis. No parent of origin effects was observed, as some favoured the expression of the paternal alleles (I.4, I.5, II.2, II.3, II.6, III.8, III.9), and others the maternal allele (II.5, II.9) $\left(\chi^{2}=2.78, P>0.05\right)$. The phase of I.2 is unknown. The F8 mutation does not appear to be influencing the XCI given that the predominantly active $\mathrm{X}$ chromosome sometimes contains the wildtype $F 8$ (II.2, II.3), sometimes the mutant F8 (I.2, I.4, II.3, II.11, III.9), and some of the females with skewed XCI are not HA carriers (I.4, II.11).

The frequency of females with dramatically skewed XCI and the overall pattern of XCI in this family were assessed. We compared the observed frequency of dramatically skewed XCI (3/11 informative females) with that predicted by 8 -cell and 16-cell models of random XCI $(0.98 / 11$ and $0.18 / 11$, respectively) and with that found in a normal population $(0.88 / 11) .{ }^{5}$ In the family presented here, there is a significantly larger proportion of females with dramatically skewed XCIR than predicted by the 8-cell or 16-cell models of random XCI, or that found in a normal population $(P<0.05, P<0.005, P<0.025$, respectively). The overall distribution of XCIR frequencies in this family was examined. Models of random XCI predict 7.5/11, 3/11, and $0.5 / 11$ females would have XCIRs falling within $1 \mathrm{SD}$ of the mean, between 1 and 2 SD, and outside 2 SD of the mean, respectively. Using the thresholds defined by the 8-cell or normal population models, 3/11 females in this family have an XCIR falling within 1 SD, 7/11 have an XCIR falling between 1 and 2 SD, and 1/11 has an XCIR falling outside 2 SD of the mean. This is significantly different from the expected distribution of XCIR frequencies $(P<0.025)$. Using the threshold values defined by the $16-$ cell model, $3 / 11,4 / 11$, and 4/11 females have XCIRs falling within 1 SD, between 1 and 2 SD, and outside 2 SD of the mean, respectively. Again, this is significantly different from expected $(P<0.005)$. Thus, using the established threshold, the number of dramatically skewed females in this family is unexpectedly high when compared with either of the two models of random XCI or observed XCIR frequencies from a normal population. Also, the distribution of XCIRs is significantly different from expected with fewer family members near the 50:50 ratio, and more than expected with higher degrees of skewing.

\section{FVIII activities correlate with the degree of XCIR skewing}

To assess whether XCIRs and the resulting proportionate inactivation of the normal $F 8$ allele could explain FVIII activity in HA carrier females, we investigated the overall relationship between $\% \mathrm{X}_{\mathrm{a}}^{\mathrm{F} 8}$ and FVIII activity. When FVIII activity was plotted against $\% \mathrm{X}_{\mathrm{a}}^{\mathrm{F} 8}$ for each $\mathrm{HA}$ carrier female, a positive linear correlation was found $\left(\mathrm{y}=0.0108 \mathrm{x}+0.0145, R^{2}=0.8657\right.$ ) (Figure 3$)$. This linear correlation supports our hypothesis that low FVIII activity is related to unfavourable XCIR skewing, which results in disproportionate representation of the mutant $F 8$ allele. The extremely low FVIII activity of III.9 (0.02) can thus be explained by her dramatically skewed XCIR $(92: 8$, SD 2$)$ in favour of the mutant F8 allele. Conversely, the heterozygous females with higher FVIII activities falling within the normal range (II.3, II.2) are skewed in favour of the normal $F 8$ allele. III.8 has a nearly 50:50 XCIR (54:46 SD 3) and correspondingly has close to half normal FVIII activity. FVIII activity is therefore not dependant on whether or not a heterozygous female's XCIR deviates from 50:50, but rather by how much has she deviated, and in which direction (ie toward or away from the expression of the $F 8$ inversion).

It is possible that additional parameters are modulating FVIII activity. One of the most important parameters is the time of day at which plasma is collected. A consistent diurnal variation in FVIII activity has been described. ${ }^{20}$ Our values, when corrected for this variation and plotted against $\% \mathrm{X}_{\mathrm{a}}^{\mathrm{F} 8}$ still showed a strong linear correlation $\left(R^{2}=0.89\right.$, data not shown).

\section{Linkage analyses at the XIC}

Some cases of familial skewed XCI have been reported to result from XIC mutations. ${ }^{21}$ In these cases, the XCIRs are completely skewed (100:0) which is not the case in the family presented here. However, XIC mutations are a formal possibility, so to determine if the skewing phenotype is linked to the XIC in this family, linkage analyses of the HUMARA marker, and three additional informative microsatellites near the XIC (DXS986, DXS1196, and DXS990) were completed on DNA from the proband (III.9), her sister (III.8), her mother (II.9), and her father (II.8) (Table 1). The mother was informative for all four markers and both the proband and her sister inherited the 


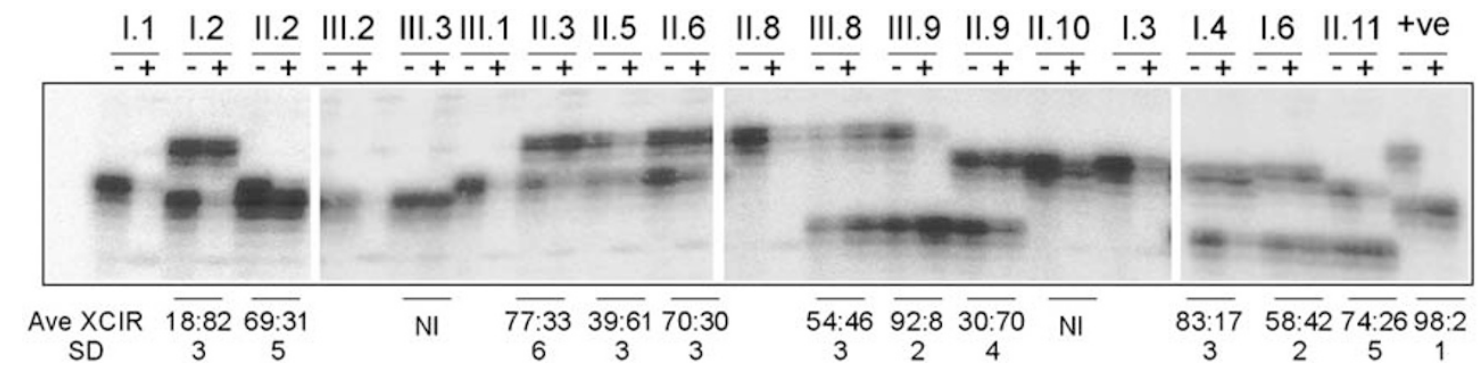

Figure 2 Three females have dramatically skewed XCIRs. The XCIR in DNA extracted from peripheral blood lymphocytes was determined by quantifying the relative methylation of the paternal and maternal androgen receptor alleles (HUMARA) $\left(X_{1}^{p}: X_{i}^{m}\right)$. This is accomplished by incubating DNA with $(+)$ or without $(-)$ a methylation sensitive endonuclease, Hhal, followed by PCR amplification of the polymorphic region of HUMARA. ${ }^{16}$ Only inactivated (methylated) alleles are undigested and therefore intact PCR templates. PCR products are separated on a polyacrylamide gel. The intensity of each allele is determined by phosphorimager. The weaker the phosphorimage signal, the more active the corresponding allele. The final paternal to maternal ratio in digested samples is normalized to the ratio in undigested samples. Representative gels are shown with the average XCIR (Ave $X_{a}^{p}: X_{a}^{m}$ ), defined as the ratio of active alleles, and SD from at least two independent experiments. Where the phase is unknown (I.2), the ratio is given arbitrarily as the larger (upper) allele: smaller (lower) allele. Negative control (-ve), no DNA. Positive control ( + ve), DNA from a female with an $\mathrm{X}$ :autosome translocation. III.3 and II.10 were non-informative (NI) at the HUMARA locus.

Table 1 Skewing phenotype not linked to the XIC

\begin{tabular}{|c|c|c|c|c|c|c|}
\hline \multirow[b]{2}{*}{ Marker } & \multirow[b]{2}{*}{ Cytogenetic location } & \multirow[b]{2}{*}{$\begin{array}{l}\text { Physical location } \\
\text { (Mbp from pter) }\end{array}$} & \multicolumn{4}{|c|}{ Participant } \\
\hline & & & II.8 (father) & III.8 (sister) & III.9 (proband) & II.9 (mother) \\
\hline XIC & Xq13 & 73 & - & - & - & - \\
\hline DXS986 & $X q 21.1$ & 79 & 162 & $162 / 166$ & $162 / 166$ & $168 / 166$ \\
\hline DXS1196 & Xq21.31 & 86 & 215 & $215 / 217$ & $215 / 217$ & $221 / 217$ \\
\hline DXS990 & $\mathrm{Xq} 21.32$ & 93 & 131 & $131 / 125$ & $131 / 125$ & $127 / 125$ \\
\hline
\end{tabular}

Mbp, mega base pairs; XIC, X-inactivation centre.

Alleles at four X-linked loci flanking the XIC are shown.

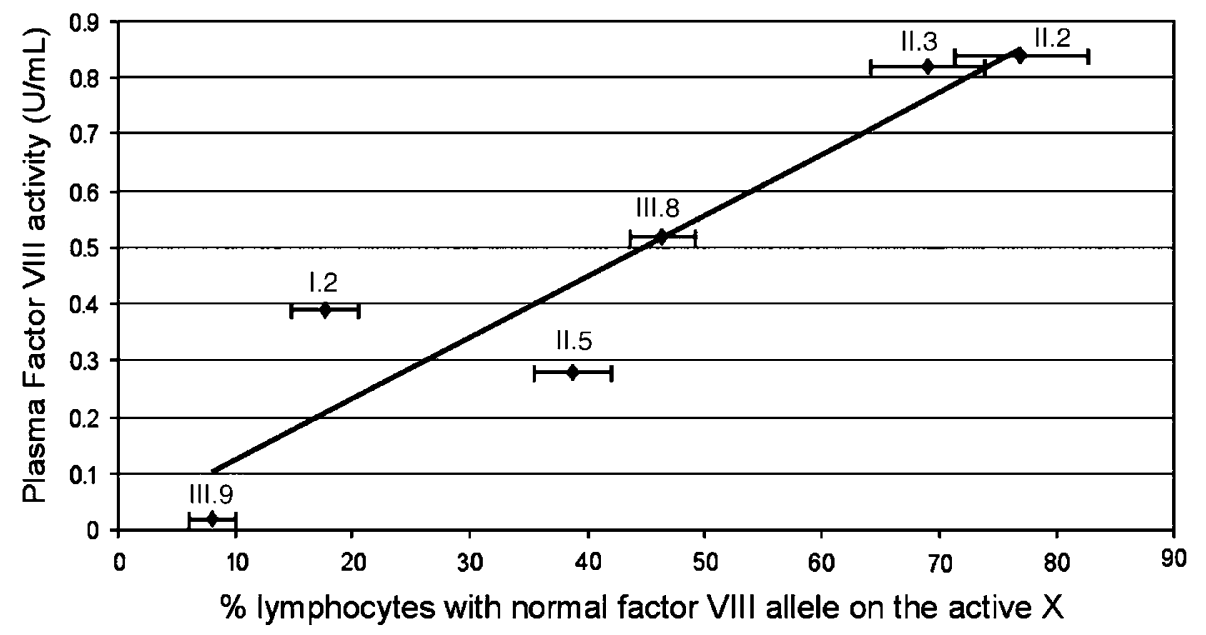

$\left(\% \mathrm{XFB}_{\mathrm{a}}\right)$

Figure 3 Factor VIII activity in heterozygous females is directly related to the percentage of lymphocytes with the normal $F 8$ allele on the active $X$ $\left(\% X_{a}^{F 8}\right)$. Results for each individual are the average $\% X_{a}^{F 8}$ from at least two independent experiments. Error bars indicate SD.

same maternal haplotype. As expected, they inherited the same paternal haplotype. Given that the sisters are divergent for the skewing phenotype, it is unlikely that XCIR skewing is linked to the XIC.

\section{Discussion}

We have described a 46, XX girl (III. 9) who is heterozygous for a $F 8$ gene inversion yet expresses severe HA with very low FVIII activity (Figure 1). Having ruled out the 
possibility that she is a compound heterozygote, we searched for an alternative explanation for her HA phenotype. We have determined that she has extreme skewing of XCI resulting in almost complete inactivation of her maternally derived $\mathrm{X}$ chromosome on which her only normal F8 gene is located (Figures 1 and 2). Two other females within the family express HA. They are heterozygous for the F8 mutation, and have skewed XCI favouring the activation of the mutant $X$ chromosome (Figure 1). Despite the fact that FVIII activity can be affected by a number of parameters (time of day, ${ }^{20}$ age, ABO blood type ${ }^{22}$ ) a strong linear correlation was observed in all HA carriers in this family between FVIII activity and the percentage of cells, which have kept the normal $\mathrm{X}$ active. These findings demonstrate that HA expression in heterozygous females in this family is due to skewed XCI.

An unexpectedly large number of females in this family were found to have dramatically skewed XCIRs (Figures 1 and 2). Furthermore, an analysis of the distribution XCI frequencies revealed fewer than expected females with XCIRs near 50:50 and more than expected females with increasingly skewed XCIRs. This distribution was significantly different than predicted by three different models that assume random XCI and suggests a genetic influence over XCI in this family.

Several genetic mechanisms that may lead to XCIR skewing have previously been described in mammals. These include chromosomal abnormalities, selection against deleterious alleles, imprinting, and mutations within the XIC such as XIST promoter mutations. In mouse, there is evidence to support a locus, the $\mathrm{X}$ controlling element (Xce), that influences the choice of which $\mathrm{X}$ will be inactivated.

We have ruled out chromosomal abnormalities as a mechanism of XCIR skewing in the proband. We have not formally determined the karyotypes of the other family members with skewed XCI; however, it is unlikely that their XCIR skewing is due to an X:autosome translocation, as none have the completely skewed XCIR expected in these translocations. Further, it is most likely that all of the skewed XCI in this family is due to a common mechanism. Therefore, we are not pursuing X:autosome translocations as a mechanism in any of the family members.

For some X-linked recessive diseases, carriers are skewed away from the expression of the mutant allele, such as in Wiskott-Aldrich Syndrome (WAS). ${ }^{23}$ Although the initial XCIR of a WAS carrier may have been unskewed, owing to a survival advantage of lymphocytes expressing the normal allele, WAS carriers have an XCIR of 100:0 in favour of the $\mathrm{X}$ chromosome with the normal allele. This extremely skewed XCIR is found only in the tissue sensitive to the disease, that is the haematopoietic cell lineages. The presented kindred does harbour an X-linked recessive disease allele, the F8 mutation. It is not likely that XCI skewing is related to the inheritance of the $F 8$ inversion, as some females have more cells expressing the normal $\mathrm{X}$, and others have more cells expressing the mutant X. Further, women from both sides of the family who have not inherited the $F 8$ mutation have somewhat or dramatically skewed XCIRs (II.6, I.2, II.11) (Figure 1). In addition, DNA for these experiments was attained from peripheral blood, and F8 mutations are not known to produce a selective disadvantage in white blood cells.

Transcription of XIST is essential for X-chromosome silencing. Mutations of the XIST promoter can completely bias XCIRs. One dominant promoter mutation prevents the $\mathrm{X}$ chromosome carrying the mutant XIST from being inactivated, ${ }^{24}$ whereas another causes the mutant $X$ to always be inactivated. ${ }^{21}$ Thus, XIST promoter mutations result in complete XCIR skewing (100:0). An XIST promoter mutation is not a likely explanation for the XCIR skewing observed in the family reported here. Barring the unlikely event of double crossovers between these tightly linked markers close to the centromere $(0.4 \%)$, the microsatellite analyses have shown that the proband and her unaffected sister (III.9 and III.8) have identical genotypes from HUMARA to DXS990 (Table 1). This suggests that they have identical XIC genotypes. As she and her sister are discordant for the skewing phenotype, it is unlikely that the XCI skewing is due to an inherited XIST mutation. A new mutation could have occurred within the XIC of III.9, although this would not explain the significant number of relatives with XCIR skewing, nor is it compatible with $<100 \%$ skewing.

Parental origin effects are unlikely to be a cause of skewing in this family. Although skewed XCI owing to imprinting has been observed in mice ${ }^{25}$ and marsupials, ${ }^{26}$ it is not believed to be involved in human XCI. ${ }^{27}$ Further, some females in this kindred have a maternally biased XCIR, and others have a paternally biased XCIR, which is inconsistent with imprinted XCI (Figure 3).

A mechanism that could result in the unusual distribution of XCIR skewing in this family that has not been ruled out by our investigations is that of a genetically influenced choice over which $\mathrm{X}$ chromosome is inactivated. Such a mechanism has been described in the mouse Xce model. ${ }^{28,29}$ Experimental observations in mice led Cattanach and Isaacson to propose that the XCIR is genetically determined by the mouse genotype at some Xce locus. They describe complementation groups, each with a unique allelic variant of the Xce, designated $\mathrm{Xce}^{\mathrm{A}} \mathrm{Xce}^{\mathrm{B}}$ $\mathrm{Xce}^{\mathrm{C}}$, and $\mathrm{Xce} \mathrm{D}^{\mathrm{D}}$. Each has a different propensity for being on the active chromosome. The final XCIR in each female mouse is determined by the difference in allele strengths of the two Xce alleles she inherited. Significant skewing will only be observed if a strong and a weak allele are both inherited.

Although mouse and human mechanisms of XCI differ in many respects, inheritance studies support the existence of a human XCE. In one study of 38 families with no 
known genetic diseases, five had familial clustering of dramatically skewed XCI. ${ }^{6}$ There was a strong sister-sister correlation and weak mother-daughter correlation of XCIR. The authors concluded that an XCE model was the only model that would adequately describe their observations. The skewed XCI trait described in that study showed linkage in the region of the XIC (Xq13-21) and to the Xq25-26 region. A recent study also describes familial skewed XCI linked to the Xq25 region in phenotypically normal females. Six out of eight females had completely skewed XCI (100:0). ${ }^{30}$ In contrast to the family we present here, an X-linked dominant inheritance was found. The authors suggest that a locus within the Xq25 region may be involved in XCI choice. If this locus acts similarly to the mouse Xce, as postulated by Naumova et al, ${ }^{6}$ perhaps this family harbours a null-mutation. This would explain the complete XCI skewing and the X-linked dominant inheritance.

The distribution of females with dramatically skewed XCIR in the family studied here is consistent with the effects predicted by the human XCE hypothesis. ${ }^{6}$ This hypothesis can explain how sib-pairs such as III.8 and III.9 can be divergent for the skewing phenotype (54:46 vs 92:8, respectively), and how a child and her grandmother, but not her mother, could be dramatically skewed, as in the case of III.9, I.4, and II.9, respectively. If XCE is X-linked, siblings could have inherited up to two XCE genotype combinations. It is formally possible that the trait is autosomal. In that case, siblings could inherit up to four combinations of XCE alleles.

We have defined two distinct phenotypes segregating in this family: the HA phenotype and the dramatically skewed XCIR phenotype. The convergence of these has led to the expression of HA in three heterozygous females. Studies are currently underway to identify chromosomal regions that may contain the putative genetic element responsible for skewed XCIR in this family.

\section{Acknowledgements}

This work was supported by grants from the CDHA, Halifax, Nova Scotia $(W G, S D)$, and the Canadian Hemophilia Society (WG, SD). NR was supported by scholarships from the Canadian Institutes of Health Research (CIHR), and the Killam Foundation. Part of this work was completed by the Australian Genome Research Facility (AGRF), Victoria, Australia. We acknowledge their contribution and the support that the AGRF receives from the Commonwealth. We thank Dr D Lillicrap and J Leggo at the National Program for Hemophilia Mutation Testing and the AHCDC for their support. We thank Dr R Howell for critical reading of the paper, Dr B Morash for technical guidance, and $P$ Steele for patient recruitment and correspondence.

\section{References}

1 Acquila M, Caprino D, Bicocchi P, Mori PG, Tagliaferri AR: A skewed lyonization phenomenon as cause of hemophilia A in a female patient. Blood 1995; 85: 599-600.
2 Bicocchi MP, Migeon BR, Pasino M et al: Familial nonrandom inactivation linked to the $\mathrm{X}$ inactivation centre in heterozygotes manifesting haemophilia A. Eur J Hum Genet 2005; 13: 635-640.

3 Lyon MF, Rastan S: Parental source of chromosome imprinting and its relevance for $\mathrm{X}$ chromosome inactivation. Differentiation 1984; 26: 63-67.

4 Penny GD, Kay GF, Sheardown SA, Rastan S, Brockdorff N: Requirement for Xist in $\mathrm{X}$ chromosome inactivation. Nature 1996; 379: $131-137$.

5 Amos-Landgraf JM, Cottle A, Plenge RM et al: X chromosomeinactivation patterns of 1,005 phenotypically unaffected females. Am J Hum Genet 2006; 79: 493-499.

6 Naumova AK, Olien L, Bird LM et al: Genetic mapping of X-linked loci involved in skewing of $\mathrm{X}$ chromosome inactivation in the human. Eur J Hum Genet 1998; 6: 552-562.

7 Maniatis T, Fritsch EF, SambrooK J: Molecular Cloning: A Laboratory Manual. Cold Spring Harbor Laboratory: Cold Spring Harbor, NY, 1982.

8 Greer WL, Lee CL, Callanan MB, Zayed E, Sadek I: Case of acute lymphoblastic leukemia presenting with $\mathrm{t}(14 ; 18) / \mathrm{BCL} 2$, $\mathrm{t}(8 ; 14) / \mathrm{cMYC}$, and $\mathrm{t}(1 ; 2) /$ FCGR2B. Am J Hematol 2003; 74 : $112-118$.

9 Ishkanian AS, Malloff CA, Watson SK et al: A tiling resolution DNA microarray with complete coverage of the human genome. Nat Genet 2004; 36: 299-303.

10 Gitschier J, Drayna D, Tuddenham EG, White RL, Lawn RM: Genetic mapping and diagnosis of haemophilia A achieved through a BclI polymorphism in the factor VIII gene. Nature 1985; 314: 738-740.

11 Rossiter JP, Young M, Kimberland ML et al: Factor VIII gene inversions causing severe hemophilia A originate almost exclusively in male germ cells. Hum Mol Genet 1994; 3: 1035-1039.

12 Sawecka J, Skulimowska J, Windyga J, Lopaciuk S, Koscielak J: Prevalence of the intron 22 inversion of the factor VIII gene and inhibitor development in Polish patients with severe hemophilia A. Arch Immunol Ther Exp (Warsz) 2005; 53: 352-356.

13 Xie YG, Zheng H, Leggo J, Scully MF, Lillicrap D: A founder factor VIII mutation, valine 2016 to alanine, in a population with an extraordinarily high prevalence of mild hemophilia A. Thromb Haemost 2002; 87: 178-179.

14 Bagnall RD, Waseem N, Green PM, Giannelli F: Recurrent inversion breaking intron 1 of the factor VIII gene is a frequent cause of severe hemophilia A. Blood 2002; 99: 168-174.

15 Williams IJ, Abuzenadah A, Winship PR et al: Precise carrier diagnosis in families with haemophilia A: use of conformation sensitive gel electrophoresis for mutation screening and polymorphism analysis. Thromb Haemost 1998; 79: 723-726.

16 Allen RC, Zoghbi HY, Moseley AB, Rosenblatt HM, Belmont JW: Methylation of HpaII and HhaI sites near the polymorphic CAG repeat in the human androgen-receptor gene correlates with $\mathrm{X}$ chromosome inactivation. Am J Hum Genet 1992; 51: 1229-1239.

17 Tilley WD, Marcelli M, Wilson JD, McPhaul MJ: Characterization and expression of a cDNA encoding the human androgen receptor. Proc Natl Acad Sci USA 1989; 86: 327-331.

18 Siegbahn A, Odlind V, Hedner U, Venge P: Coagulation and fibrinolysis during the normal menstrual cycle. Upsala J Med Sci 1989; 94: $137-152$.

19 Waters JJ, Campbell PL, Crocker AJ, Campbell CM: Phenotypic effects of balanced X-autosome translocations in females: a retrospective survey of 104 cases reported from UK laboratories. Hum Genet 2001; 108: 318-327.

20 Iversen PO, Groot PD, Hjeltnes N, Andersen TO, Mowinckel MC, Sandset PM: Impaired circadian variations of haemostatic and fibrinolytic parameters in tetraplegia. Br J Haematol 2002; 119: $1011-1016$.

21 Plenge RM, Hendrich BD, Schwartz C et al: A promoter mutation in the XIST gene in two unrelated families with skewed X-chromosome inactivation. Nat Genet 1997; 17: 353-356.

22 Orstavik KH, Magnus P, Reisner H, Berg K, Graham JB, Nance W: Factor VIII and factor IX in a twin population. Evidence for a 
major effect of ABO locus on factor VIII level. Am I Hum Genet 1985; 37: 89-101.

23 Greer WL, Kwong PC, Peacocke M, Ip P, Rubin LA, Siminovitch $\mathrm{KA}$ : X-chromosome inactivation in the Wiskott-Aldrich syndrome: a marker for detection of the carrier state and identification of cell lineages expressing the gene defect. Genomics 1989; 4: $60-67$.

24 Tomkins DJ, McDonald HL, Farrell SA, Brown CJ: Lack of expression of XIST from a small ring X chromosome containing the XIST locus in a girl with short stature, facial dysmorphism and developmental delay. Eur J Hum Genet 2002; 10: 44-51.

25 West JD, Frels WI, Chapman VM, Papaioannou VE: Preferential expression of the maternally derived $\mathrm{X}$ chromosome in the mouse yolk sac. Cell 1977; 12: 873-882.
26 Sharman GB: Late DNA replication in the paternally derived X chromosome of female kangaroos. Nature 1971; 230: 231-232.

27 Zeng SM, Yankowitz J: X-inactivation patterns in human embryonic and extra-embryonic tissues. Placenta 2003; 24: $270-275$.

28 Cattanach $\mathrm{BM}$, Isaacson JH: Controlling elements in the mouse X chromosome. Genetics 1967; 57: 331-346.

29 Chadwick LH, Willard HF: Genetic and parent-of-origin influences on $\mathrm{X}$ chromosome choice in Xce heterozygous mice. Mamm Genome 2005; 16: 691-699.

30 Cau M, Addis M, Congiu R et al: A locus for familial skewed $\mathrm{X}$ chromosome inactivation maps to chromosome Xq25 in a family with a female manifesting Lowe syndrome. J Hum Genet 2006; 51: 1030-1036. 